\title{
Mode Coupling Analysis of Hollow Ring-Core Fibers for OAM Transmission
}

\author{
M. Lonardi ${ }^{(1)}$, G. Guerra(2) , L. Marcon(2), R. M. Nejad ${ }^{(3)}$, M. Santagiustina ${ }^{(2)}$,
} A. Galtarossa ${ }^{(2)}$, L. A. Rusch ${ }^{(3)}$, A. Bononi ${ }^{(1)}$ and L. Palmieri ${ }^{(2, *)}$

(1) Department of Information Engineering, University of Parma, Italy

(2) Department of Information Engineering, University of Padova, Italy, ("luca.palmieri@unipd.it)

(3) Department of Electrical and Computer Engineering, Laval University, Québec, Canada

Abstract We present an analytical and numerical description of coupling between OAM modes in hollow ring-core fibers affected by stress birefringence and ellipticity. The analysis paves the way to a better modeling of propagation in these fibers.

\section{Introduction}

Mode division multiplexing (MDM) can greatly expand the capacity of future optical transmission systems, however, complex receiver-side multiple-input, multiple-output (MIMO) processing is required in most demonstrated linearly polarized MDM systems. Orbital angular momentum (OAM) modes for MDM have the potential for MIMO-free or reduced-MIMO signal processing. In fibers where the effective indices of OAM modes of different order are sufficiently separated, crosstalk among OAM modes is low. ${ }^{1,2}$ Air core fibers support a high number of OAM modes due to the large refractive index contrast at the air/glass interface, with air core OAM fibers supporting 12 and 36 OAM modes demonstrated. . $^{3,4}$ Recently MIMO-free data transmission over a $1.2 \mathrm{~km}$ air core fiber supporting 12 OAM modes was demonstrated. ${ }^{5}$

Crosstalk induced by coupling among propagating OAM modes constrains capacity increases. Coupling in propagation could be the result of physical perturbations such as fiber core ellipticity, birefringence, mechanical stress, micro bending, etc. The design and fabrication of fibers with minimum OAM mode coupling requires an understanding of the impact of these coupling mechanisms. Current statistical models describing propagation impairments in non-ideal fibers are limited to the strong mode coupling regime and provide only asymptotic results. ${ }^{6}$ A numerical study showed these asymptotic properties will be observed for few mode fiber only after thousands of kilometers. ${ }^{7}$ These models are therefore doubly inappropriate for OAM fibers optimized for minimum coupling, and designed for short reach applications. In this paper, we examine mode coupling in hollow ring-core (air core) OAM fibers to develop models for mode propagation. We discuss the interactions among OAM modes, as well as their interactions with parasitic TE and TM modes. In particular, we study the effect of fiber core ellipticity and birefringence, and we discuss coupling among modes of different order due to each of these impairments.

\section{Composition of OAM modes}

Propagation in any cylindrical fiber with an arbitrary number of concentric rings of homogeneous lossless material is described by the complete set of hybrid (or vector) modes, $\mathrm{TE}_{0, p}, \mathrm{TM}_{0, p}, \mathrm{HE}_{\ell, p}$ and $\mathrm{EH}_{\ell, p}$, where $\ell \neq 0$ and the arbitrary sign of $\ell$ corresponds to the 2-fold degeneracy of $\mathrm{HE}$ and $\mathrm{EH}$ modes. ${ }^{8}$ With respect to basis of circular polarizations, the electric field of hybrid modes can be expressed as

$$
\begin{aligned}
& \boldsymbol{E}=\left[f_{\ell}(r)+j g_{\ell}(r)\right] e^{j(\ell+1) \varphi} \hat{R}+ \\
& \quad\left[f_{\ell}(r)-j g_{\ell}(r)\right] e^{j(\ell-1) \varphi} \hat{L}+j h_{\ell}(r) e^{j \ell \varphi} \hat{z},
\end{aligned}
$$

where $f_{\ell}, g_{\ell}$ and $h_{\ell}$ are mode-dependent real functions, $\hat{z}=(0,0,1)$, and $\hat{R}=(1,-j, 0) / \sqrt{2}$ and $\hat{L}=(1, j, 0) / \sqrt{2}$ represent right- and left-handed circular polarization respectively. This expression shows that the transverse field of hybrid modes is a combination of two OAM "beams" of orders $\ell+1$ and $\ell-1$. In weakly-guiding fibers one of the two "beams" becomes negligible; therefore, hereinafter we call dominant the transverse component that survives in the weak guidance limit and secondary the transverse component that would not be present in a weakly-guiding fiber, but does exist in OAM fiber with strong index contrast. Note that also the longitudinal component tends to be negligible in the weak guidance limit.

When expressed as in (1), hybrid modes are directly related to OAM modes; so according to the customary definition, ${ }^{3}$ we can write

$$
\begin{array}{lll}
\mathrm{OAM}_{ \pm n, p}^{ \pm} \triangleq \mathrm{HE}_{ \pm(n+1), p} & \text { for } & n \geq 0 \\
\mathrm{OAM}_{ \pm n, p}^{\mp} \triangleq \mathrm{EH}_{ \pm(n-1), p} & \text { for } & n \geq 2 .
\end{array}
$$

This definition is based on the dominant component of the field; the secondary component is considered a spurious term of an otherwise pure OAM mode. ${ }^{9}$ While TE and TM modes are not 


\begin{tabular}{|c|c|c|c|c|c|c|}
\cline { 2 - 7 } \multicolumn{1}{c|}{} & $O A M_{+n}^{+}$ & $O A M_{-n}^{-}$ & $O A M_{+m}^{-}$ & $O A M_{-m}^{+}$ & $T E$ & $T M$ \\
\hline$\hat{R}$ & $n+2$ & $-n$ & $m$ & $-m+2$ & 1 & 1 \\
\hline$\hat{L}$ & $n$ & $-n-2$ & $m-2$ & $-m$ & -1 & -1 \\
\hline$\hat{z}$ & $n+1$ & $-n-1$ & $m-1$ & $-m+1$ & -- & 0 \\
\hline
\end{tabular}

Tab. 1: Orders and strength of the components of OAM, TE and TM modes $(n \geq 0, m \geq 2)$. White, pink and green cells indicate the dominant, secondary and longitudinal

components of the field, respectively. Some indices are omitted for brevity.

useful in OAM-multiplexed transmission systems, these modes must be taken into account in the coupled mode analysis as long as they can propagate. It is also worthwhile to identify dominant and secondary components by their azimuthal order; this can be done by comparison with LP modes in the weak guidance limit and the result is summarized in Tab. 1.

\section{Coupled Mode Analysis}

According to coupled mode theory, ${ }^{10}$ the complex amplitudes of the $N$ propagating modes, represented by the vector $c$, vary along $z$ as

$$
\frac{d \boldsymbol{c}}{d z}=-j(\boldsymbol{\beta}+\boldsymbol{K}) \boldsymbol{c},
$$

where $\boldsymbol{\beta}=\operatorname{diag}\left(\beta_{1}, \ldots, \beta_{N}\right)$ is the diagonal matrix of propagation constants and the $N \times N$ matrix $\boldsymbol{K}(z)$ represents the coupling per unit length. For small perturbation the elements of $K$ are ${ }^{10}$

$$
K_{\mu, \nu}=\omega \int_{0}^{\infty} \int_{0}^{2 \pi} r \boldsymbol{E}_{\mu}^{*} \tilde{\boldsymbol{\epsilon}} \boldsymbol{E}_{\nu} d \varphi d r=K_{\nu, \mu}^{*},
$$

where $r$ and $\varphi$ are the polar coordinates, $\tilde{\epsilon}$ is the $3 \times 3$ matrix of dielectric perturbation, and the 3 dimensional vectors $\boldsymbol{E}_{\mu}$ and $\boldsymbol{E}_{\nu}$ are the electric fields of modes $\mu$ and $\nu$, properly normalized. ${ }^{10}$

The complete evaluation of the overlap integral (5) is generally quite involved; however, at least the integration with respect to $\varphi$ can be performed easily. ${ }^{11,12}$ Actually, in almost every case of practical interest the elements of the dielectric perturbation matrix $\tilde{\boldsymbol{\epsilon}}(r, \varphi)$ can be expressed as the product of two functions each depending on either $r$ or $\varphi$. Moreover, since $\tilde{\boldsymbol{\epsilon}}(r, \varphi)$ has to be singlevalued, the dependence on $\varphi$ can be expressed by terms like $e^{j k \varphi}$, where $k=0, \pm 1, \ldots$ are the azimuthal orders of the perturbation. Given that the fields have a similar dependence on $\varphi$ [see (1)], the integration over $\varphi$ is nonzero only if specific "resonances" occur. ${ }^{11}$ Specifically, let $n_{\mu}$ be the azimuthal order of a component of mode $\boldsymbol{E}_{\mu}$ and similarly let $n_{\nu}$ be the azimuthal order of a component of $\boldsymbol{E}_{\nu}$. Then, we can say that these two modes can couple through the two respective components only if

$$
n_{\mu}-n_{\nu}= \pm k,
$$

where the minus sign in the right term stems from the fact that one of the fields in (5) is conjugated.

\begin{tabular}{|l|c|c|c|c|}
\cline { 2 - 5 } \multicolumn{1}{c|}{} & Air hole & Ring core & Trench & Cladding \\
\hline Radii $[\mu \mathrm{m}]$ & $\leq 9.1$ & $\leq 11.3$ & $\leq 16.2$ & $\leq 25.0$ \\
\hline Refr. Indx. & 1.000 & 1.473 & 1.438 & 1.444 \\
\hline
\end{tabular}

Tab. 2: Parameters of the simulated fiber.

Note that condition (6) is necessary but not sufficient for coupling to occur, because in some cases (5) can be zero after integration over $r$. Moreover, as clarified in the following, the structure of $\tilde{\epsilon}$ selects which field components can originate the coupling.

We start considering stress birefringence. With respect to the basis of circular polarizations, the corresponding dielectric perturbation is ${ }^{12}$

$$
\tilde{\boldsymbol{\epsilon}} \approx \varepsilon_{c o} \Delta n\left(\begin{array}{lll}
0 & 1 & 0 \\
1 & 0 & 0 \\
0 & 0 & 0
\end{array}\right),
$$

where $\varepsilon_{c o}$ is the dielectric permittivity of the core and $\Delta n$ the birefringence. The azimuthal order is $k=0$, hence coupling may occur only between components of the same azimuthal order $\left(n_{\mu}=\right.$ $n_{\nu}$ ) and, given the structure, only between orthogonally polarized transverse components $(\hat{R} \leftrightarrow \hat{L})$. Consulting Tab. 1 we can conclude, for example, that modes $\mathrm{OAM}_{-m, p}^{+}$and $\mathrm{OAM}_{m, p}^{-}$cannot couple because of birefringence; differently, coupling may occur between modes $\mathrm{OAM}_{m, p}^{+}$and $\mathrm{OAM}_{m, p}^{-}$, through their dominant components, and between modes $\mathrm{OAM}_{m, p}^{+}$and $\mathrm{OAM}_{m+4, p}^{-}$, through the secondary ones.

To verify these conclusions, we numerically evaluated the coupling coefficients (5) for a hollow ring-core fiber supporting (at $1550 \mathrm{~nm}$ ) 26 information bearing modes and 2 parasitic TE/TM modes; ${ }^{3}$ the parameters are given in Tab. 2; field distributions have been calculated with ComSol ${ }^{\mathrm{TM}}$. The resulting coupling coefficients for birefringence are reported in Fig. 1, normalized to the largest one; for this case the strongest coupling occurs between modes $\mathrm{OAM}_{ \pm 6}^{ \pm}$and $\mathrm{OAM}_{ \pm 6}^{\mp}$ and the respective coefficient is about $\left(2.9 \times 10^{6}\right) \Delta n \mathrm{rad} / \mathrm{m}$. The dots reported on the figure are the couplings predicted by the above theoretical analysis. As we see there is complete agreement, that is, all non-zero values are anticipated by theory.

In the case of slight ellipticity in a cylindrical fiber made of $M$ concentric homogeneous rings, the dielectric perturbation is given by ${ }^{12}$

$$
\tilde{\boldsymbol{\epsilon}}=\varepsilon_{0}\left[\sum_{k=1}^{M-1} \gamma_{k}\left(n_{k}^{2}-n_{k+1}^{2}\right) \delta\left(r-r_{k}\right)\right] \cos 2 \varphi,
$$

where $r_{k}$ is the ideal outer radius of the $k$ th ring, $n_{k}$ its refractive index and $\gamma_{k} \geq 0$ is the maximum radius variation. The dielectric perturbation is scalar, hence coupling can occur only between 

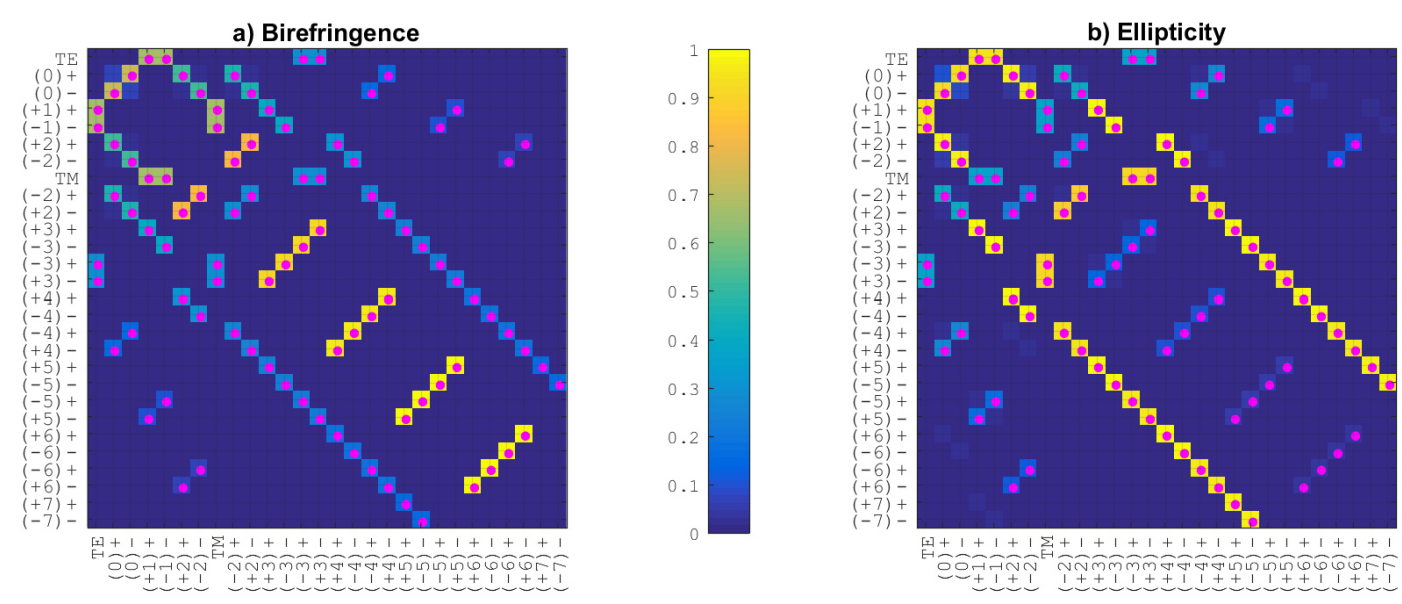

Fig. 1: Normalized magnitudes of coupling coefficients for (a) birefringence and (b) ellipticity, numerically evaluated for the fiber described in Tab. 2. Dots mark coupling predicted by theory. TE/TM modes are of order $(0,1)$; " $( \pm k) s$ " indicates $\mathrm{OAM}_{ \pm k, 1}^{s}$.

equal polarizations of the transverse components $(\hat{R} \leftrightarrow \hat{R}, \hat{L} \leftrightarrow \hat{L})$ or between longitudinal components $(\hat{z} \leftrightarrow \hat{z})$, and has azimuthal order $k=2$ $\left(n_{\mu}-n_{\nu}= \pm 2\right)$. For example, from Tab. 1 we see that these conditions allow coupling between $\mathrm{OAM}_{n, p}^{-}$and $\mathrm{OAM}_{-m+2, p}^{+}$only for $n=m=2$. Most importantly, there is always strong coupling between OAM modes of the same kind, but with orders $\pm n$ and $\pm(n+2)$.

Also in this case we performed the numerical analysis, assuming $\gamma_{k}=\gamma$ for all interfaces; results are shown in Fig. 1 and are normalized to the coupling coefficients between modes $\mathrm{OAM}_{ \pm 4}^{ \pm}$ and $\mathrm{OAM}_{ \pm 2}^{ \pm}$; the respective coefficient is about $\left(1.25 \times 10^{4}\right) \gamma \mathrm{rad} / \mathrm{m}$ (with $\gamma$ expressed in meters). As for previous case there is complete agreement with the theoretical analysis.

Quite surprisingly, birefringence and ellipticity give the same coupling although with different strengths. It can be shown that this is a consequence of the fact that both cause coupling only between hybrid modes whose orders $\ell_{1}$ and $\ell_{2}$ are such that $\left|\ell_{1}-\ell_{2}\right|=2$.

\section{Conclusions}

The analysis presented here is a preliminary but promising step towards a better description and deeper understanding of coupling mechanism in ring-core fibers for OAM transmissions, such as those observed experimentally between TE/TM and $\mathrm{OAM}_{ \pm 1} \cdot{ }^{13}$ The tables shown in Fig. 1 provide rich quantitative information about coupling mechanisms, some of which, like the critical coupling with TE and TM modes, have not been discussed due to the limited space. This kind of analysis is fundamental for the assessment of the effect of mode coupling, which also depends on the difference between the propagation constants of the modes and the distance of propagation. Further analyses to include these aspects as well as the other sources of coupling (such as bending or twisting) are needed.

M.L. and A.B. acknowledge the European
Commission (project ROAM, Horizon 2020) for financial support.

\section{References}

[1] N. Bozinovic, Y. Yue, Y. Ren, M. Tur, P. Kristensen, H. Huang, A. E. Willner, and S. Ramachandran, "Terabitscale orbital angular momentum mode division multiplexing in fibers," Science 340, 1545-1548 (2013).

[2] R. M. Nejad, K. Allahverdyan, P. Vaity, S. Amiralizadeh, C. Brunet, Y. Messaddeq, S. LaRochelle, and L. A. Rusch, "Mode division multiplexing using orbital angular momentum modes over 1.4-km ring core fiber," Journal of Lightwave Technology 34, 4252-4258 (2016).

[3] C. Brunet, P. Vaity, Y. Messaddeq, S. LaRochelle, and L. A. Rusch, "Design, fabrication and validation of an OAM fiber supporting 36 states," Optics Express 22, 26117 (2014).

[4] P. Gregg, P. Kristensen, and S. Ramachandran, "13.4 km OAM state propagation by recirculating fiber loop," Optics Express 24, 18938-18947 (2016).

[5] K. Ingerslev, P. Gregg, M. Galili, F. Da Ros, H. Hu, F. Bao, M. A. U. Castaneda, P. Kristensen, A. Rubano, L. Marrucci et al., "12 Mode, MIMO-free OAM transmission," in "Optical Fiber Communication Conference," (Optical Society of America, 2017), pp. M2D-1.

[6] K.-P. Ho and J. M. Kahn, "Linear propagation effects in mode-division multiplexing systems," Journal of Lightwave Technology 32, 614-628 (2014).

[7] F. Ferreira, S. Sygletos, and A. Ellis, "Impact of linear mode coupling on the group delay spread in few-mode fibers," in "Optical Fiber Communications Conference and Exhibition (OFC), 2015," (IEEE, 2015), p. Tu2D.1.

[8] C. G. Someda, Electromagnetic waves (CRC/Taylor \& Francis, Boca Raton, FL, 2006).

[9] L. Wang, A. Corsi, L. A. Rusch, and S. LaRochelle, "Investigation of orbital angular momentum mode purity in air-core optical fibers," in "2016 IEEE Photonics Society Summer Topical Meeting Series (SUM)," (2016), pp. 203-204.

[10] D. Marcuse, "Coupled-mode theory for anisotropic optical waveguides," Bell System Technol. J. 54, 985-995 (1975).

[11] L. Palmieri, "Coupling mechanism in multimode fibers," in "Photonics West OPTO," vol. 9009 (SPIE, San Francisco (US-CA), 2014), vol. 9009, pp. 90090G-90090G-9.

[12] L. Palmieri and A. Galtarossa, "Coupling effects among degenerate modes in multimode optical fibers," IEEE Photonics Journal 6, 1-8 (2014).

[13] R. M. Nejad, L. Wang, J. Lin, S. Larochelle, and L. A. Rusch, "Parasitic effect of TE and TM modes in OAMMDM transmission systems," in "CLEO Conference," (Optical Society of America, 2017). 